\title{
ELEMENTOS CLAVES PARA FOMENTAR LA INVESTIGACIÓN EN LAS UNIVERSIDADES EN EL BICENTENARIO DEL PERÚ
}

\section{KEY ELEMENTS TO ENCOURAGE RESEARCH IN UNIVERSITIES, IN}

\section{THE BICENTENNIAL OF PERU}

(D) Omar Bullón Solís ${ }^{*}$, (D) Fiorella Rocío Valero Palomino ${ }^{1}$

omar.bullon@unsch.edu.pe; fiorella.valero@unsch.edu.pe

${ }^{1}$ Universidad Nacional de San Cristóbal de Huamanga, Ayacucho, Perú

*Correspondencia: Omar Bullón Solís. Email: omar.bullon@unsch.edu.pe

Recibido: 30.06.21 | Aprobado: 15.07.21

\section{RESUMEN}

El artículo presenta cuatro elementos claves que se considera relevantes para fomentar la investigación en las universidades, sin pretender señalar que son los únicos. Se menciona primero desarrollar competencias para la investigación y luego fomentar las redes de trabajo colaborativo; segundo, el fortalecimiento del liderazgo de los centros de investigación en las universidades, tercero, al uso de las TIC en el desarrollo y distribución de la investigación; y cuarto, a fomentar la ética y los valores en la actividad investigativa. Se plantea que estos elementos deben conjugarse con las políticas institucionales vigentes en las universidades e instituciones vinculadas a la investigación. Estas políticas adquieren un protagonismo clave en el fomento de la investigación motivo por lo cual se presenta el presente análisis que conmemora el bicentenario de la independencia del Perú.

Palabras clave: Pilares, desarrollo, investigación, universidades.

\begin{abstract}
The article presents four elements that are considered relevant for promoting research at universities, without claiming to be the only ones. The first is to develop research skills and then to foster collaborative networks teams; second, the strengthening of the leadership of research centers in universities; third, the use of ICTs in the development and distribution of research; the fourth, the promotion of ethics and values in research activity. It is suggested that these elements should be combined with the institutional policies in force in universities and institutions linked to research. These policies acquire a key role in the promotion of research, which is why this analysis is presented to commemorate the bicentennial of the independence of Peru.
\end{abstract}

Keywords: pillars, development, research, universities. 


\section{INTRODUCCIÓN}

En la actualidad, la producción científica se emplea para medir cuanto aporte al conocimiento genera un país y este está vinculado con su desarrollo social y económico. En este campo, el Perú muestra progresos en la última década como lo demuestra el crecimiento del 88\% de la productividad científica entre los años 2012 y 2017 (CONCYTEC, 2019); sin embargo, este desarrollo no es suficiente para igualar las cifras de producción científica de otros países como México, Chile o Argentina. Esto se debe a desbalances notorias en el aporte de las regiones del Perú. Se resalta que la ciudad de Lima llega a concentrar al 79\% de toda la actividad científica a nivel nacional (CONCYTEC, 2019), teniendo en cuenta este resultado, se aprecia una desproporción con la producción científica de las demás regiones, con quienes el desarrollo de la ciencia puede triplicarse a nivel nacional, luego de fortalecer sus instituciones dedicadas a la investigación.

El desarrollo de las actividades investigativas conforma una parte importante del desarrollo económico de un país. Es pieza fundamental para mejorar la productividad y conseguir dar un mejor uso de los recursos. En una sociedad globalizada, la productividad de las empresas debe ser innovadora, creativa y con capacidad de resolver problemas; pero en muchas ocasiones se encuentran sin la capacidad técnica, o sin los recursos para poder invertir en desarrollar la investigación. Por este motivo, es relevante considerar la colaboración entre el sector empresarial y las universidades para concretar los campos de la investigación vinculados a resolver dificultades productivas y económicas.

De esta manera, en la actualidad existe una demanda social para incrementar la productividad científica que actualmente se encuentra en un nivel bajo. Es allí donde se evidencia la debilidad de la capacidad investigadora de las universidades. Con lo cual se hace evidente la necesidad de una reforma en los centros de investigación y en las universidades (Bautista-Vallejo et al., 2020).

En este recorrido es cuando llega la cuarta revolución industrial. Esta mega tendencia llega con nuevas técnicas de producción que incluye sistemas inteligentes integrados, con gran capacidad productiva que puede representar un verdadero peligro para los países que se encuentran en vía de desarrollo. El peligro surge por la incapacidad de estos últimos de adaptarse a los modernos modelos productivos que les supera en adaptación a la capacidad técnica y los vuelve consumidores dependientes de estas nuevas tecnologías de producción. Frente a esta dificultad los investigadores cumplen el rol de ser intermediarios en el proceso de transferencia tecnológica y en la adaptación de las tecnologías adquiridas a un contexto empresarial o social a nivel local.

Por lo tanto, las actividades vinculadas a fomentar la investigación deben fortalecerse y cumplir su papel relevante en la sociedad moderna. Se requiere que los centros de investigación participen en la adquisición, la transformación y la comunicación, tanto para la generación de nuevas tecnologías como para la adaptación de estas a los entornos locales. Es conocido que las tecnologías pueden tener efecto positivo o negativo de acuerdo cómo se utilice y para darle un buen uso, se requiere de investigar su aplicación.

La interconexión actual representa una oportunidad para el surgimiento de centros de investigación que tengan conexión con otros centros tecnológicos a nivel mundial, de donde se intercambia el conocimiento y las experiencias acumulados (Antúnez-Sanchez 
\& Veytia-Bucheli, 2020). En otras palabras, fomentar la investigación permite a los investigadores a estar prestos para percibir riesgos del futuro, colaborar con el desarrollo del país y generar innovación en el progreso local; pero su desarrollo requiere estar basados en algunos pilares; por lo tanto ¿Cuáles son estos elementos claves para fomentar la investigación en las universidades y centros de investigación en las regiones?

Esta contribución apunta a captar la atención de autoridades de instituciones públicas y educativas sobre la importancia de desarrollar la investigación en las universidades y en otros centros de investigación a nivel nacional. La propuesta de cuatro elementos claves para su desarrollo forma parte de una base horizontal sobre la cual las instituciones puedan organizar sus propias políticas, con enfoque claro, liderazgo y dirección que generen la productividad científica requerida. La tecnología de la información juega un rol importante en este desarrollo y es una oportunidad para gestionar las relaciones entre los investigadores (Bautista-Vallejo et al., 2020).

Se resalta que, en el proceso de generar conocimiento en las universidades y centros de investigación, se requiere un comité de ética que regule la forma y los medios en los que se desarrolla la producción científica. El objetivo es guiar el trabajo de forma transparente y honesta (Cruz-Ortiz et al., 2020). Establecer un código de ética tiene una importancia directa con el centro que acoge las investigaciones y con la misma investigación en sí. Establecer los principios éticos que guían la investigación resulta una pieza fundamental para fomentar su desarrollo debido que aporta credibilidad y confianza a los resultados publicados producto de las investigaciones.

\section{DESARROLLO}

\section{Primer elemento: Desarrollar competencias para la investigación}

La competitividad en la investigación está referido al fortalecimiento de conocimientos y habilidades de las personas que realizan actividades científicas. El desarrollo de estas capacidades es fundamental para el éxito de una investigación, debido que en estas radica la importancia de iniciar y culminar un proceso investigativo sin complicaciones durante su desarrollo u observaciones posteriores. Tal es el caso que las instituciones que financian las investigaciones exigen la revisión de competencias técnicas y científicas para la evaluación de los objetivos de los proyectos de producción científica (SENESCYT, 2019), motivo por el cual diversas investigaciones no logran obtener financiamiento para su desarrollo.

Por lo tanto, las capacidades humanas representan un componente clave para la realización de proyectos de investigación. Es fundamental que las universidades y centros de investigación doten al a cargo de la investigación, con las competencias requeridas para desarrollar sus estudios científicos.

Formar investigadores es un proceso complejo que responde al desarrollo de habilidades estratégicas de investigación. Realizar esta formación es estratégico debido que lamentablemente el capital humano vinculado a la producción científica en las universidades está disminuyendo en los países en vías de desarrollo. Un efecto de la falta de apoyo gubernamental y la captación de talento por parte de los países más desarrollados (Reynosa-Navarro et al., 2020). 
Por consiguiente, la formación de competencias para la investigación requiere ser un eje principal en las universidades y en los centros de investigación; y está vinculada con la formación docente debido que es el elemento que por su naturaleza de trabajo debe realizar investigación (Gómez-Arteta et al., 2020). Actualmente la sociedad necesita de producción científica que permita solucionar los problemas de su entorno próximo (Reynosa-Navarro et al., 2020) y la universidad dispone como capital humano a sus docentes quienes deben desempeñar las labores investigativas.

Se hace mención que en el presente aquellos profesionales con competencias para investigar se encuentran bajo una presión cada vez mayor por parte de sus centros de investigación para publicar hallazgos permanentemente, casi en base a una agenda de entregas. Esto ocurre porque las universidades están presionadas a difundir resultados para demostrar niveles de calidad basado en estadísticas; por lo que aquellos con competencias investigativas son exigidos a cumplir estándares de producción científica. Ante esta circunstancia de presión para la presentación y publicación de resultados, existe la posibilidad que estos se encuentren fuera del marco ético o se enfoquen en lograr una meta cuantitativa, es decir, producir cantidad en lo más simple y no en lo más relevante (Cruz-Ortiz et al., 2020).

Por lo tanto, la universidad y otros centros de investigación presentan, en las condiciones descritas anteriormente, una contradicción, cuando se piensa en desarrollar la función de investigar y proponer exigencia de producción con tiempos predeterminados. De esta forma, existe una tendencia a la preponderancia del docente investigador sobre el docente impartidor de conocimientos en el aula de clase, con todavía un desconocido efecto de esta preponderancia en la calidad educativa. Se viene valorando las capacidades investigativas por sobre las habilidades pedagógicas de los docentes (Bautista-Vallejo et al., 2020).

\section{Segundo elemento. Fortalecer el liderazgo en los centros de investigación}

La universidad y los centros de investigación públicos y privados son las instituciones encargadas de promover, orientar y dar a conocer los resultados de las investigaciones, por lo cual cumplen un rol primordial en la producción científica. Estas instituciones se concentran en producir y expandir el desarrollo del conocimiento científico (Aguilar de Cáceres, 2020). Al respecto, el sector privado en el Perú, como también en la mayoría de los países latinoamericanos, todavía no desarrolla una inversión significativa en cuanto a investigación. Por tanto, el rol principal para desarrollar investigación recae en las universidades y centros de investigación, dada su naturaleza y misión el cual es promover la investigación y para lograrlo se requiere de fortalecer el liderazgo.

El objetivo de estas instituciones científicas es alcanzar la capacidad de poder ser entes generadores de conocimiento, al tiempo que su producción científica contribuya al desarrollo de la ciencia. Para alcanzar estos objetivos, las universidades y los centros de investigación requieren de una organización que propicie el trabajo colaborativo por lo cual debe motivar y generar el liderazgo de quienes tienen a su cargo los proyectos de investigación. 
Es primordial contar con instituciones que lideren la investigación. Para lograr ello se requiere establecer ciertas políticas que guíen el desarrollo. En la figura 1 se sugiere cuatro políticas vinculadas a las líneas de investigación, al establecimiento de modelos formativos, orientación de la investigación y la socialización de las líneas investigativas.

\section{Figura 1}

Políticas sugeridas en los centros de investigación para promover la investigación

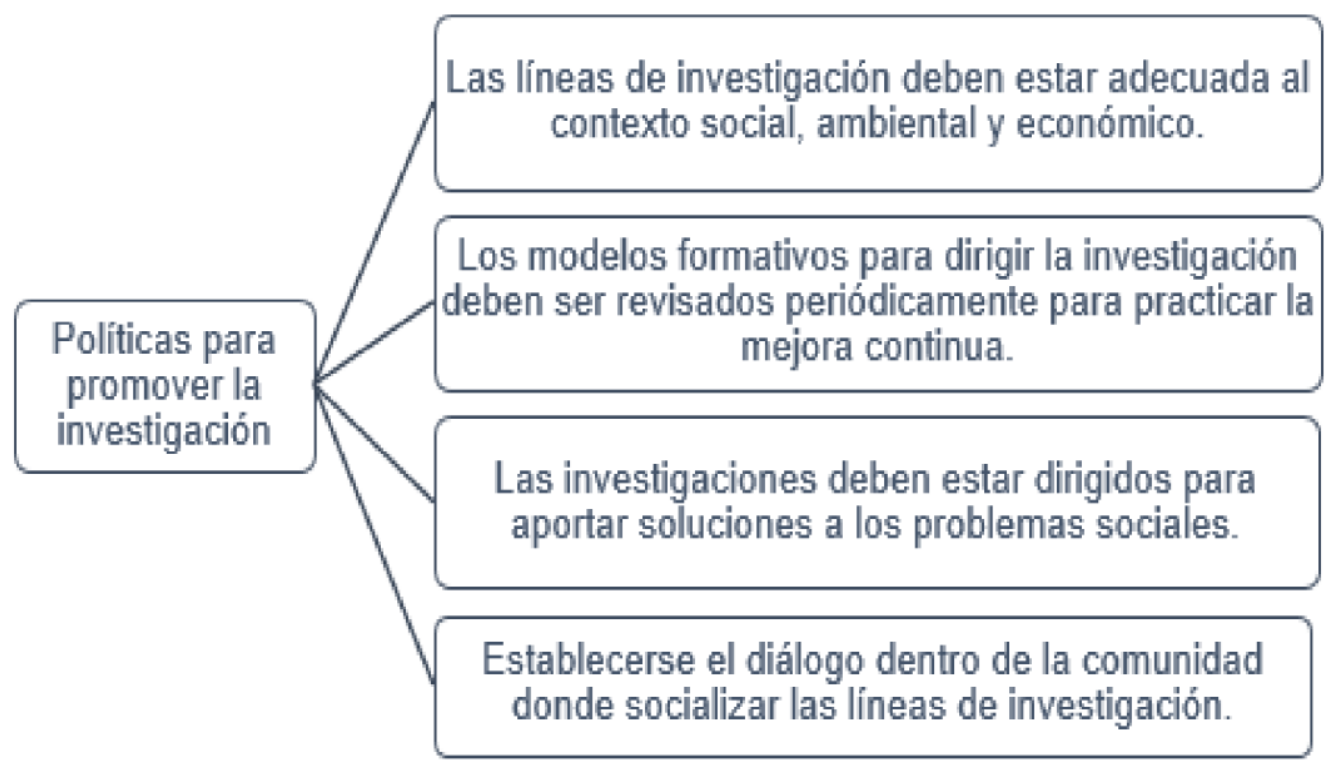

Fuente: Elaboración propia.

Se reconoce que las universidades tienen la misión de generar investigación en diversos campos que respondan al contexto socioeconómico y ambiental. Para alcanzar esta misión se requiere de contar con capital humano con la capacidad para realizarlo y de entes rectores que promuevan. De ahí surge la necesidad de contar con un liderazgo que dirija y motiva el desarrollo de investigación. Se requiere consolidar el liderazgo para diseñar y consolidar estrategias institucionales que produzcan conocimiento y desarrollen transferencia tecnológica en beneficio de su sociedad. En este camino resulta primordial que las instituciones promuevan una gestión eficiente y efectiva en cuanto al manejo de los recursos disponibles para promover y generar investigación.

Las instituciones promotoras de la investigación requieren promover contar con personal idóneo para realizar investigación. El personal docente de las universidades requiere tener una libertad para investigar basada en la guía de un sustento epistemológico y científico. Esta base es la que proporciona la capacidad de investigar sobre líneas de interés para la sociedad y es el liderazgo de las autoridades lo que lo proporciona (GómezArteta et al., 2020).

Es relevante que las autoridades a cargo de estas instituciones deban desarrollar criterios idóneos para la contratación, renovación y promoción del personal que labora en los campos de investigación. Gran parte del éxito de la producción científica se puede atribuir a las habilidades de dirección y manejo de las autoridades de estas instituciones (Perines, 2020). 
Por otra parte, se menciona también que las autoridades a cargo deben realizar la gestión necesaria para proteger la propiedad intelectual existente durante la investigación (SENESCYT, 2019). Se requiere liderazgo para gestionar el conocimiento que se genera en estas instituciones educativas. La publicación de las investigaciones requiere de experiencia en el registro, publicación y patentamiento de servicios resultantes de la actividad investigativa.

Es cuando se afirma que el grado de liderazgo en las instituciones refleja el nivel de madurez que estas tienen y su capacidad para contribuir con la sociedad. Las autoridades a cargo juegan un rol importante y la gestión y control de los procesos vinculados a la investigación, requieren de una dirección constante y firme para producir conocimiento.

\section{Tercer elemento: Usar las TIC para el desarrollo multidisciplinario de la investigación}

Las TIC conocidas como las tecnologías de la información y la comunicación están presentes en el nuevo escenario de la globalización. Además, el uso de estas herramientas ha sido reforzadas en su uso debido a la expansión de todo tipo de equipamiento relacionado con la conectividad, el $4 \mathrm{G}$ y ahora el $5 \mathrm{G}$ que ha llevado a acceder a la información a nuevos niveles (Hernández-Palma et al., 2020). Para tener buenos profesionales, hoy en día se requiere que conozca del uso de instrumentos vinculados a las TIC, tanto para la conexión y trabajo colaborativo, como también para dar a conocer su trabajo.

El uso de estas herramientas se encuentra hoy vinculado también con las bibliotecas. Estos espacios de almacenamiento y difusión de contenido han ido evolucionando para servir de fuente para las actividades investigativas. El servicio de las bibliotecas requiere de expandirse del entorno físico hacia entornos virtuales (AntúnezSanchez \& Veytia-Bucheli, 2020).

En este cambio adaptativo las bibliotecas cumplen un nuevo rol clave en el desarrollo de la investigación, particularmente las relacionadas con las investigaciones del tipo revisiones sistemáticas. Este tipo de investigación requiere conexión a grandes bases de datos que sin las TIC sería imposible contar con toda la información.

Es conocido que los avances de las TIC en los servicios de información para bibliotecas académicas es un recurso relevante que fomenta la investigación y contribuye a mejorar la calidad de los resultados (Hernández-Palma et al., 2020). Estas tecnologías permiten el acceso a las bibliotecas de instituciones de renombre, así como la creación de una red para conectarse con otros investigadores. Las universidades deben promover su implementación y formación en el uso de estas tecnologías en la investigación.

Desarrollar las TIC en las universidades y centros de investigación genera un acceso rápido y seguro a una ingente cantidad de información presente en la nube a través de las bases de datos indizadas. Esto genera una aceleración en el desarrollo de la investigación. Además, facilita la creación de redes de investigación a nivel global, que tienden a estimular significativamente el progreso de la investigación en las universidades. Además, adquirir estas tecnologías son relativamente de fácil acceso. La principal dificultad es 
contar con personal con las habilidades adecuadas para promover su uso y fomentar el hábito en su manipulación que estas herramientas requieren.

\section{Figura 2}

Requerimientos para hacer a las bibliotecas a los usuarios.

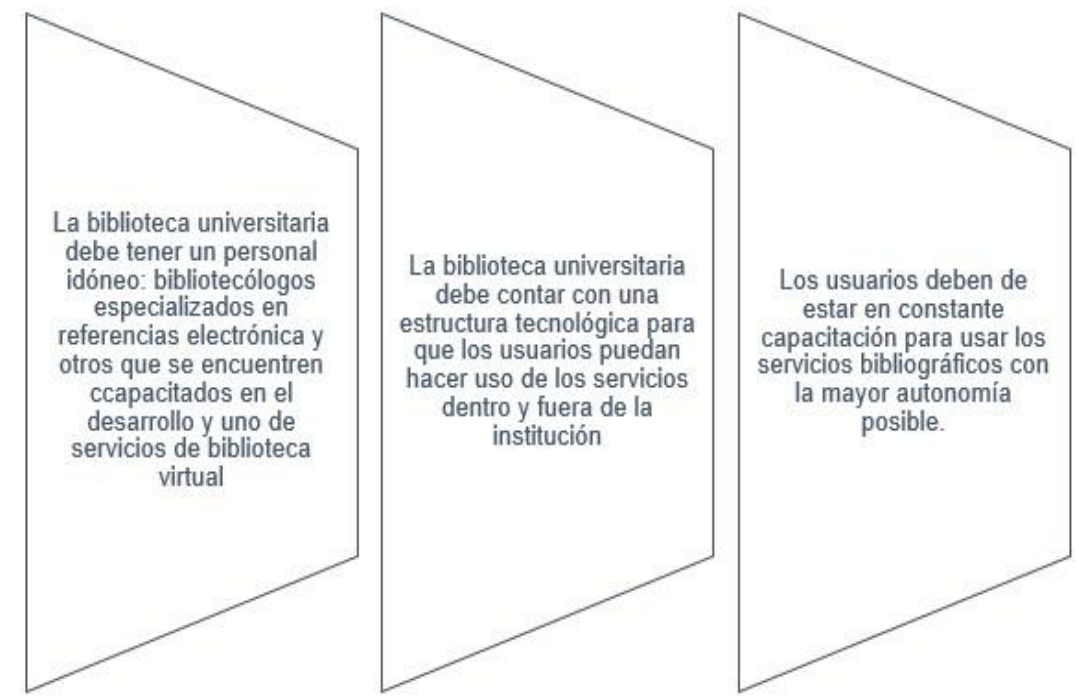

Fuente: Hernández-Palma, Niebles-Núñez, Pacheco-Ruiz (2020).

Con el objetivo de promover el uso de las TIC se requiere instaurar el proceso de gestión de la información como una actividad estratégica en los centros de investigación. En las universidades, es necesario desarrollar estos procesos de forma y que está relacionada con la experiencia de quienes participan en actividades de investigación (Antúnez-Sanchez \& Veytia-Bucheli, 2020). La investigación es un proceso de generación de nuevos conocimientos y es necesario el almacenamiento de lo logrado. Los medios de almacenamiento digital vinculado a las TIC son ideales para esto debido a su conveniencia y accesibilidad.

Se reconoce que las TIC están creando una verdadera revolución en todos los ámbitos. Ha cambiado radicalmente muchas áreas del desarrollo de la investigación, donde se incluye acceso a bases de información en entornos sistematizados (BautistaVallejo et al., 2020). Por lo tanto, se afirma que las TIC deben verse como una herramienta que debe utilizarse de forma continua en los centros de investigación. La adaptación a los avances tecnológicos es fundamental para quienes realizan la labor de investigadores.

\section{Cuarto elemento: Promover las implicancias éticas en la investigación}

La promoción de la ética en la investigación es un estatus importante que requiere un énfasis en los centros de investigación. Está vinculado a la formación de una cultura de intercambio (Antúnez-Sanchez \& Veytia-Bucheli, 2020) donde la producción de conocimiento debe interactuar con otros, teniendo en cuenta los códigos de ética profesional.

La ética comienza con el diseño de líneas de investigación. El dilema de decidir qué línea es relevante para aportar conocimiento genera un conflicto ético que las universidades deben priorizar (Cruz-Ortiz et al., 2020). Una práctica que, si bien parece 
simple y común, puede requerir un análisis complejo y una buena ayuda para justificarla. Esta actividad es vital para promover la investigación en las universidades.

La investigación se guía por principios éticos. Representan los límites entre lo permitido y lo que está fuera de lo posible y su aplicación está vinculada a las buenas prácticas de investigación. Aunque se refieren al diseño del estudio y a la población, los códigos éticos pueden validar o cancelar un procedimiento de puesta en marcha (CuadrosContreras, 2019).

El componente ético se ha convertido en un eje fundamental que requiere tenerse en cuenta cuando se desarrolla investigación. Es necesario establecer principios claros a través de reglas debidamente socializadas para su aplicación. La ética es necesaria para la formación de futuros investigadores. No debería verse como una simple regulación normativa, sino como una forma respetable de realizar ciencia.

Se llega a afirmar que la ética no es sólo un código o regulación del procedimiento, sino debemos comprender a la ética como una práctica innata asociada al ejercicio de la auto regulación de nuestras acciones y deseos. (Estrada-Cely \& Parra-Herrera, 2016)suponen dos tipos de consideraciones particulares, las de tipo intraespecífico, reconocidas por diversos autores del campo de la Bioética como bioética intraespecífica, microbioética o bioética deontológica, basada en el principio de igualdad y cuya prima facie de responsabilidad reposa en el respeto a la autonomía y la no maleficencia, dentro de las que se estructuran las relaciones entre los seres humanos o personas; y las de tipo interespecífico, constituida para las relaciones establecidas entre los seres humanos y las demás especies vivientes, basadas en el principio de desigualdad y guiadas por los principios de justicia y beneficencia desde la premisa del principio de responsabilidad, dado el superior carácter del ser humano en su condición único ser ético y moral 1, 2, 5, 6, 7, 9, 10 . Implicaciones éticas y bioéticas intraespecíficas Las investigaciones que involucren seres humanos como participantes en experimentos, encuestas, entrevistas y estudios cualitativos, requieren un marco ético estricto que garantice la veracidad de los resultados y su legalidad, sin vulnerar los derechos y principios de los involucrados. Como implicaciones éticas intraespecíficas generales se considera que todos los seres humanos nacen libres y con los mismos derechos, por lo que deben ser tratados con idéntico respeto, fraternidad y dignidad. Estos principios consignados en la Declaración Universal de los Derechos Humanos de 1948, deberán guiar la totalidad de investigaciones que impliquen seres humanos. En correspondencia con la Declaración de Helsinki de 1964 y los principios éticos universales, los participantes en una investigación tienen los siguientes derechos: - Recibir información adecuada acerca de los objetivos, métodos, fuentes de financiamiento, posibles conflictos de intereses, afiliaciones institucionales del investigador, beneficios calculados, riesgos previsibles e incomodidades derivadas del experimento.

No puede existir la investigación sin tener en cuenta la ética. La ética es parte del proceso de investigación y ayuda a fortalecer el vínculo de respeto y aprecio entre los investigadores. Mejora la imagen de la investigación en sí y respalda la institución que la sustenta y promueve. 


\section{CONCLUSIÓN}

En el desarrollo de este artículo se presentó cuatro elementos claves para fomentar la investigación en las universidades y centros de investigación. Con esto se busca que estas instituciones promuevan la investigación a nivel regional, con la finalidad de superar la barrera de producción científica donde se afirma que el $80 \%$ proviene de la ciudad de Lima (CONCYTEC, 2019).

El primer elemento fue desarrollar las competencias para la investigación. La formación y desarrollo de las habilidades investigadoras en los centros de investigación es un elemento primordial para cumplir la misión de las universidades el cual es generar conocimiento e innovación para resolver los problemas de su sociedad (Blanco-Guzmán, 2020). Para hacer esto, las estrategias deben enfocarse en desarrollar capacidades investigativas en el personal involucrado con esta actividad. Estas habilidades comienzan con el dominio del método científico, así como con la práctica en el uso de tecnologías y dominio de las metodologías de investigación; sin descuidar la ética y los valores asociados al desarrollo de la investigación. Lograr estas capacidades permiten a los investigadores construir una cultura científica en sintonía con la ciencia y motivarlos a abordar problemas complejos.

El segundo elemento fue fortalecer el liderazgo de los centros de investigación. Existe una necesidad actual por impulsar la gestión eficiente en las universidades y centros de investigación. Para ello se requiere generar liderazgo en las autoridades que conduzcan a incentivar la investigación activa de las personas involucradas con el desarrollo de la investigación. El liderazgo es requerido también para promocionar las líneas de investigación y su actualización periódica. Se requiere de una gestión transparente por parte de las autoridades para recoger los problemas sociales y económicos de la comunidad y brindar las pautas para desarrollar la investigación. Además, es requerido un liderazgo adecuado para diseñar programas investigativos sin presionar con resultados rápidos y poco no relevantes al personal a cargo de llevar a cabo las investigaciones.

Tercero, el uso de las TIC en el desarrollo y distribución de la investigación. La tecnología TIC ofrece ventajas competitivas en cuanto a la rapidez con la que se obtiene la información. Un hecho notable es que las bibliotecas en la actualidad están en proceso de convertirse en centros de información digital que facilita el acceso a los investigadores (Hernández-Palma et al., 2020). Estos medios digitales son ahora una fuente relevante para promover la investigación. Las universidades y centros de investigación necesitan de estos espacios para adquirir y difundir conocimientos. La adquisición e implementación de las TIC es necesaria como un conjunto de herramientas relacionadas con la creación de vínculos con el mundo y facilitar el desarrollo de la investigación.

Y cuarto, fomentar la ética y los valores en la actividad investigativa. Poner énfasis en la ética es fundamental particularmente en la formación de nuevos investigadores. La adherencia a las pautas éticas no solo garantiza la capacidad técnica de llevar a cabo un proyecto de investigación; sino que, también contribuye directamente a la implementación exitosa de las soluciones exitosas, debido que mantienen una reputación positiva (EstradaCely \& Parra-Herrera, 2016) suponen dos tipos de consideraciones particulares, las de tipo intraespecífico, reconocidas por diversos autores del campo de la Bioética como 
bioética intraespecífica, microbioética o bioética deontológica, basada en el principio de igualdad y cuya prima facie de responsabilidad reposa en el respeto a la autonomía y la no maleficencia, dentro de las que se estructuran las relaciones entre los seres humanos o personas; y las de tipo interespecífico, constituida para las relaciones establecidas entre los seres humanos y las demás especies vivientes, basadas en el principio de desigualdad y guiadas por los principios de justicia y beneficencia desde la premisa del principio de responsabilidad, dado el superior carácter del ser humano en su condición único ser ético y moral 1, 2, 5, 6, 7, 9, 10 . Implicaciones éticas y bioéticas intraespecíficas Las investigaciones que involucren seres humanos como participantes en experimentos, encuestas, entrevistas y estudios cualitativos, requieren un marco ético estricto que garantice la veracidad de los resultados y su legalidad, sin vulnerar los derechos y principios de los involucrados. Como implicaciones éticas intraespecíficas generales se considera que todos los seres humanos nacen libres y con los mismos derechos, por lo que deben ser tratados con idéntico respeto, fraternidad y dignidad. Estos principios consignados en la Declaración Universal de los Derechos Humanos de 1948, deberán guiar la totalidad de investigaciones que impliquen seres humanos. En correspondencia con la Declaración de Helsinki de 1964 y los principios éticos universales, los participantes en una investigación tienen los siguientes derechos: • Recibir información adecuada acerca de los objetivos, métodos, fuentes de financiamiento, posibles conflictos de intereses, afiliaciones institucionales del investigador, beneficios calculados, riesgos previsibles e incomodidades derivadas del experimento. Es importante no separar la ética de los procesos de investigación, debido que son la fuerza principal detrás de los resultados obtenidos y publicados.

\section{REFERENCIAS BIBLIOGRÁFICAS}

Aguilar de Cáceres, M. C. (2020). Gestión estratégica en los centros de investigación de la Universidad Nacional de Asunción. Población y Desarrollo, 26(51), 20-25. https://doi.org/10.18004/pdfce/2076-054x/2020.026.51.020

Antúnez-Sanchez, A. G., \& Veytia-Bucheli, M. G. (2020). Desarrollo de competencias investigativas y uso de herramientas tecnológicas en la gestión de información. Revista Conrado, 16(72), 96-102. http://scielo.sld.cu/scielo.php?script=sci_arttext\&pid=S1990-86442020000100096

Bautista-Vallejo, J. M., Duarte de Krummel, M., Hernández-Carrera, R. M., \& EspigaresPinazo, M. J. (2020). Investigar en la Universidad hoy. Apuntes para el liderazgo educativo y el cambio social. Revista Científica de La UCSA, 7(1), 39-48. https://doi.org/10.18004/ucsa/2409-8752/2020.007.01.039-048

Blanco-Guzmán, M. (2020). Desarrollo de competencias básicas de investigación. Ajayu Órgano de Difusión Científica Del Departamento de Psicología UCBSP, 18(1), 2551. http://www.scielo.org.bo/scielo.php?script=sci_arttext\&pi d=S207721612020000100002\&lang=pt $\% 0 \mathrm{~A} \quad$ http://www.scielo.org.bo/pdf/rap /v18n1/v18n1_a02.pdf 
CONCYTEC. (2019). Principales indicadores bibliométricos de la actividad científica peruana 2012-2017 (CONCYTEC (ed.); Primera ed). Consejo Nacional de Ciencia Tecnología e Innovación Tecnológica.

Cruz-Ortiz, M., Pérez-Rodríguez, M. del C., Jenaro-Rio, C., Flores-Robaina, N., \& TorresApolo, V. A. (2020). Ethical implications for research: A constant defiance in a changing world. Horizonte Sanitario, 19(1), 9-17. https://doi.org/10.19136/ hs.a19n1.3277

Cuadros-Contreras, R. (2019). Ética y formación de investigadores: La importancia de las virtudes y la sabiduría práctica. Revista Colombiana de Educación, 1(79),

237-238. https://doi.org/10.17227/rce.num79-7972

Estrada-Cely, G. E., \& Parra-Herrera, J. P. (2016). Las implicaciones éticas y bioéticas en la investigación científica. CES Medicina Veterinaria y Zootecnia, 11(2), 115-118.

https://doi.org/10.21615/cesmvz.11.2.9

Gómez-Arteta, I., Sánchez-Rossel, M., \& Bonifaz-Valdez, B. (2020). Percepción de docentes universitarios sobre la investigación y libertad de cátedra. Aportes de La Comunicación y $\quad$ La Cultura, 28(1), 45-53. http://www.scielo.org.bo/scielo.php?script=sci_arttext\&pid=S230686712020000100006

Hernández-Palma, H. G., Niebles-Nuñez, W., Pacheco-Ruiz, C., \& Rojas-Martínez, C. (2020). Technological strategies at university libraries as a resource center for research and learning in the Colombian Caribbean region. Formacion Universitaria,

13(6), 51-60. https://doi.org/10.4067/S0718-50062020000600051

Perines, H. A. (2020). La formación en investigación educativa de los futuros profesores. Formación Universitaria, 13(4), 139-152. https://doi.org/10.4067/s071850062020000400139

Reynosa-Navarro, E., Serrano-Polo, E., Ortega-Parra, A., Navarrol-Silva, O., CruzMontero, J., \& Salazar-Montoya, E. (2020). Estrategias didácticas para investigación científica: relevancia en la formación de investigadores. 21(1), 1-9.

SENESCYT. (2019). Propuesta de metodología para la determinación del índice de competitividad. https://siau.senescyt.gob.ec/imagenes/2020/10/Informecompetitividad.pdf

\section{CITAR COMO:}

Bullón Solís, O., \& Valero Palomino, F. R. (2021). Elementos claves para fomentar la investigación en las universidades en el bicentenario del Perú. Puriq, 3(3), 366-376. https://doi.org/10.37073/puriq.3.3.207 